FORMATION Formation emploi

Revue française de sciences sociales

125 | Janvier-Mars 2014

Pêle-Mêle

\title{
Le rôle de la concurrence dans l'essor des écoles supérieures de commerce
}

The role of competition in the development of French business schools

Die Rolle der Konkurrenz im Aufschwung der Business Schools

El papel de la competencia en el auge de las escuelas superiores de comercio

\section{Marianne Blanchard}

\section{(2) OpenEdition}

Journals

Édition électronique

URL : http://journals.openedition.org/formationemploi/4124

DOI : 10.4000/formationemploi.4124

ISSN : 2107-0946

\section{Éditeur}

La Documentation française

Édition imprimée

Date de publication : 25 mars 2014

Pagination : 7-28

ISSN : 0759-6340

\section{Référence électronique}

Marianne Blanchard, «Le rôle de la concurrence dans l'essor des écoles supérieures de commerce », Formation emploi [En ligne], 125 | Janvier-Mars 2014, mis en ligne le 31 mars 2016, consulté le 30 octobre 2020. URL : http://journals.openedition.org/formationemploi/4124 ; DOI : https://doi.org/ 10.4000/formationemploi.4124 


\title{
Le rôle de la concurrence dans l'essor des écoles supérieures de commerce
}

\begin{abstract}
Marianne Blanchard Maître de conférence en sociologie à l'ESPE (Ecole supérieure du professorat et de l'éducation) Midi-Pyrénées (université Toulouse 2), rattachée au CERTOP (Centre d'étude et de recherche, travail, organisation, pouvoir).
\end{abstract}

Résumé

Le rôle de la concurrence dans l'essor des écoles supérieures de commerce

Cet article analyse les transformations qu'ont connues les écoles supérieures de commerce, en France, depuis la fin des années 1990. A travers l'étude de trois processus (internationalisation, développement de la recherche et diversification de l'offre de formation et du recrutement) il s'agit de mettre en évidence les logiques qui président à ces transformations. L'enjeu est notamment de montrer que si les demandes externes (demandes des familles ou des entreprises) peuvent jouer un rôle, celui-ci peut apparaître secondaire face aux logiques d'imitation et de concurrence à l'œuvre dans l'espace où évoluent ces établissements.

Mots clés : grande école, étude historique, concurrence, activité de recherche

Abstract

The role of competition in the development of French business schools

This article focuses on the recent transformations of some French business schools since the late 1980s. Logics behind these evolutions are questioned through the analysis of three processes (internationalization, development of research activities, diversification of both the offered training programs and recruitment paths). More precisely, if external demands (from the families and the business sphere) are important factors, our goal here is to show the preeminence of imitation and competition logics in the evolution of these schools.

Keywords : elite school, historic study, competition, research

Journal of Economic Literature: $\mathbf{J} 23$

Traduction : Auteur 
En 2012-2013, les écoles de commerce ont accueilli 133800 étudiants, soit 5,6 \% des inscrits dans l'enseignement supérieur français. Si cette proportion peut apparaitre relativement modeste, il faut rappeler qu'au début des années 1980, elle ne dépassait pas les $2 \%$, et qu'il y a encore dix ans, elle n'était que de 3,5\%. Ces écoles ont de fait connu, au cours de la dernière décennie, une croissance sans précédent. Cette évolution " quantitative " s'est accompagnée de modifications profondes dans l'organisation même de la plupart de ces établissements, qu'il s'agisse de l'offre de formation, des modalités de recrutement ou encore de la constitution du corps enseignant.

Notre propos est ici d'éclairer les transformations qui ont marqué, depuis la fin des années 1990, ce secteur relativement peu étudié de l'enseignement supérieur, en se focalisant plus particulièrement sur les écoles supérieures de commerces (ESC). Par ce terme, on désigne les établissements reconnus par l'Etat, habilités à délivrer le grade de master, pour la plupart étroitement liés à une chambre de commerce, et qui recrutent une partie de leurs étudiants en classes préparatoires. La délimitation d'un tel sous-groupe d'étude apparaît nécessaire ; en effet, le ministère de l'Éducation nationale recensait plus de 200 écoles de commerce à la rentrée 2007. Or derrière ce titre générique d'école de commerce se cache une grande variété d'établissements ; il est donc difficile de parler "des écoles de commerce " en général, tant les trajectoires, les niveaux de formation, le statut juridique ou encore le mode de recrutement varient d'une institution à l'autre. C'est pour cette raison que l'on distingue les ESC d'autres établissements, considérant qu'au-delà de leurs différences, des tendances communes émergent dans leurs évolutions récentes.

Cet article interroge en particulier les logiques à l'œuvre derrière ces évolutions, en montrant qu'elles ne peuvent se réduire à une réponse à des demandes externes, émanant des étudiants et de leurs familles ou des entreprises. Plus précisément, sans nier le rôle joué par ces demandes, on insistera ici sur les logiques propres à l'espace concurrentiel dans lequel évoluent ces établissements.

Les écoles que nous désignons ici sous le terme d'ESC ne sont pas les seules à dispenser des formations à la gestion en France : aux autres écoles de commerce s'ajoutent les formations universitaires, dans les IUT (institut universitaire de technologie), les IAE (Institut d'administration des entreprises), mais aussi dans certaines STS (Section de technicien supérieur) et dans les IEP (Institut d'études politiques).

L'espace des formations à la gestion en France apparaît de fait comme « composite et hétérogène ". Il tend à de nombreux égards à fonctionner comme un marché (de Saint Martin, 1997), plus précisément comme un "marché-jugement» au sens de L. Karpik (1989). 
En effet, l'offre de formation à la gestion se diversifie essentiellement par sa " qualité "; le choix des étudiants dépend alors pour partie du jugement qu'ils se feront de cette qualité, en s'appuyant sur des réseaux d'informations et notamment sur des " prescripteurs ", comme les revues ou encore les pouvoirs publics ${ }^{1}$.

Dès lors, les établissements de formation vont chercher à agir sur les représentations des étudiants, en s'efforçant d'améliorer leur réputation et leur statut. Cette lutte de statut passe par un travail de validation par la sphère économique. En effet, dans la mesure où les attentes des étudiants intègrent des considérations en termes de débouchés sur le marché du travail, les établissements doivent aussi s'assurer de la reconnaissance de leurs diplômes par les entreprises. Néanmoins, le statut reflète aussi des éléments propres à la sphère académique, comme le type et le niveau de recrutement (Bourdieu, 1989; Pavis, 2003a).

Cette concurrence de statut touche particulièrement les ESC, dans la mesure où leurs ressources dépendent essentiellement des frais de scolarité et donc du recrutement d'étudiants ${ }^{2}$.

Cette concurrence donne lieu à la fois à des logiques de segmentation et de convergence au sein de l'espace des ESC. Segmentation, car à la façon des entreprises étudiées par le sociologue américain H. White (1981), les écoles ne cherchent pas tant à éliminer leurs concurrentes qu'à se situer dans une "niche ", correspondant à une certaine qualité de " produit » et attirant un certain type de " clientèle ». Convergence, car afin d'améliorer leur statut, les responsables des écoles tendent à prendre pour modèle celles qu'ils perçoivent comme étant les plus légitimes - ce que P. DiMaggio et W. Powell (1983) qualifient d'isomorphisme "mimétique» - tout en se conformant à des normes édictées par des instances extérieures, comme l'Etat (isomorphisme coercitif).

Nous analyserons ici trois de ces formes de convergence. En premier lieu, il s'agira d'étudier des évolutions généralement regroupées sous le terme d' "internationalisation ", comme celles liées à la volonté d'être reconnu au-delà des frontières nationales. Dans un deuxième temps, on considèrera le " tournant académique " que ces écoles ont pris, depuis la fin des années 1990. On constate en effet, au cours des deux dernières décennies, un développement sans précédent des activités de recherche au sein des ESC, et, corollairement, une transformation extrêmement rapide du corps enseignant. Enfin, on analysera le double processus de diversification de l'offre de formation (multiplication des programmes) et du recrutement.

1. Pour partie seulement, car des mécanismes de sélection scolaire entrent en jeu. Plus généralement, les choix des étudiants sont en partie déjà largement informés par leurs trajectoires scolaires et sociales.

2. Une étude de l'ACFCI (Assemblée des chambres françaises de commerce et d'industrie) réalisée auprès de 28 écoles montre que, en 2009-2010, les frais de scolarités représentaient en moyenne 59 \% de leurs ressources (http://www.esc.fr/les-esc-en-chiffres). Toujours selon cette étude, les chambres de commerces fournissent $11 \%$ des ressources, la taxe d'apprentissage $10 \%$, la formation continue $8 \%$, les collectivités territoriales $3 \%$, pour les $10 \%$ restants, les apports sont variés (investissements, fondations, etc.). 
Ces trois transformations sont présentées de façon distincte mais sont en réalité interdépendantes et apparaissent étroitement liées à la concurrence de statut qui oppose les écoles. Ainsi la multiplication des formations - à dimension nationale ou internationale et l'élargissement du recrutement peuvent s'expliquer pour partie par la nécessité d'engranger plus de frais de scolarité et d'atteindre une taille critique afin de pouvoir recruter plus d'enseignants et financer leurs activités de recherches. Réciproquement, l'essor des activités de recherche et les transformations du corps professoral résultent pour partie d'une volonté de se conformer à des standards internationaux, dans le but d'attirer plus d'étudiants - français ou étrangers.

Cet article est issu d'une thèse soutenue à l'automne 2012, qui analyse l'évolution d'un groupe d'ESC dans une perspective socio-historique, de la fin du XIXe siècle à 2010. Ce travail s'est essentiellement concentré sur des ESC de province : ESC Amiens, ESC Bordeaux, ESC Brest, ESC Clermont-Ferrand, ESC Dijon, ESC Grenoble, ESC Le Havre, ESC Lyon, ESC Marseille, ESC Montpellier, ESC Nantes, ESC Nice, ESC Pau ESC Poitiers, ESC Reims, ESC Rennes, ESC Rouen ${ }^{3}$, mais il a aussi intégré HEC (Hautes études commerciales), l'ESSEC (Ecole supérieure des sciences économiques et commerciales), toutes deux situées en région parisienne, ainsi que l'ESC Paris. Concernant une période plus restreinte, l'article s'appuie sur trois sortes de données : des données quantitatives (statistiques produites par les ministère de l'Education nationale et de l'Enseignement supérieur et de la recherche; données de divers rapports - AERES - Agence d'évaluation de la recherche et de l'enseignement supérieur -, chambres régionales des comptes ; données disponibles dans la presse), des entretiens auprès de responsables et personnels d'établissements (41 entretiens dans 14 établissements différents auprès de directeurs, directeurs de programmes, responsables administratifs - service relation international, relations avec les entreprises - et enseignants) et enfin l'analyse de documents publiés par les écoles ellesmêmes (plaquettes publicitaires, sites Internet, chartes communes etc.).

\section{L'internationalisation des Ecoles supérieures de commerce}

Le premier phénomène de convergence entre les ESC analysées dans cet article concerne leur « internationalisation ». Ce terme recouvre en réalité une pluralité de mesures ; néanmoins, deux dimensions centrales du phénomène d' "internationalisation " des écoles de commerce peuvent être distinguées : celle concernant le contenu des formations et visant

3. Le choix de ces établissements s'explique par leur histoire commune : les plus anciens sont régis, à partir de 1947, par une réglementation commune qui organise notamment les modalités de recrutement des élèves et de délivrance du diplôme qui leur est commun. En 1965, ces écoles prennent le nom d'ESCAE (Ecoles supérieures de commerce et d'administration des entreprises). Le cadre qui les régit s'assouplit au cours des décennies suivantes et disparait en 1991. Pour plus d'informations, voir thèse de l'auteur (Blanchard, 2012). 
à fournir une " culture internationale » aux étudiants, et celle consistant à faire reconnaître un établissement donné hors des frontières nationales. Alors que la première dimension émerge dans les années 1970, c'est la seconde dimension qui devient centrale dans les années 2000. C'est également dans cette période que les accréditations internationales se diffusent en France, contribuant à la fois à servir et à accélérer l'internationalisation des écoles.

\subsection{L'internationalisation des ESC, un phénomène polymorphe et relativement ancien}

Enseigner les langues étrangères ou en anglais, doter les étudiants d'une " culture internationale ", faciliter les séjours à l'étranger, attirer des étudiants internationaux, mettre en place des doubles diplômes, recruter des enseignants étrangers, ouvrir des programmes hors des frontières : aussi diverses soient-elles, ces mesures peuvent être considérées comme participant de l' "internationalisation" des écoles supérieures de commerce. Ainsi, le terme recouvre des processus multiples, qui renvoient aussi bien au contenu qu’à l'organisation de la formation.

Phénomène polymorphe, l'« internationalisation » des ESC est aussi un phénomène ancien qui a pu recouvrir une ampleur et des significations différentes au cours du temps. Dès la création des premières écoles à la fin du XIXe siècle, leurs promoteurs insistent sur la nécessité de dispenser une formation internationale aux futurs commerçants. Néanmoins, ce n'est qu'à partir des années 1970 que les responsables des écoles se montrent réellement soucieux de doter les étudiants en « compétences internationales ". À la fin de cette décennie, HEC et l'ESCP ouvrent leurs premières filières et options " internationales ". La mobilité des étudiants devient également un enjeu et plusieurs ESC incitent leurs étudiants à effectuer des stages à l'étranger. Dès 1977, l'ESC Nantes, qui apparaît ici parmi les pionnières, envoie l'ensemble de ses étudiants en deuxième année effectuer un séjour d'études sur un campus américain Au cours des années 1980, le phénomène s'accélère et les ESC insistent davantage sur l'apprentissage de plusieurs langues étrangères, et incluent des cours à dimension internationale (affaires internationales, "interculturalité », etc.). En outre, la majorité des écoles étudiées établissent alors des accords d'échanges avec des établissements étrangers. On pourrait ainsi multiplier les exemples de création de filières internationales, ou d' "instituts " chargés, si ce n'est de favoriser les échanges internationaux au sein des écoles, du moins d'afficher leur "bonne volonté " internationale : en 1983, l'ESC Lyon crée une filière " Japon » et une association composée de grandes entreprises "dont le but est de favoriser la formation d'étudiants dotés d'un profil international " ; en 1986 c'est l'ESC Poitiers qui met en place l'Institut de développement des relations internationales, afin de favoriser les échanges d'étudiants et d'enseignants ; et en 1989,

4. Source : plaquette publicitaire pour l'ESC Lyon, 1983. 
l'ESC Nantes inaugure un Institut des relations internationales, chargé de coordonner les accords internationaux de l'établissement ${ }^{5}$.

Ce souci croissant de l'international peut résulter des évolutions du système économique. En effet, les échanges avec les pays étrangers augmentent de façon régulière à partir des années 1960, et particulièrement avec les pays européens, alors que la construction de la communauté économique s'accélère. Les écoles répondraient alors à une demande des entreprises pour des cadres dotés de " compétences internationales ", manifestant ainsi leur proximité avec les milieux économiques.

Pourtant, il serait trompeur de ne voir dans cette évolution des écoles de commerce qu'une réponse mécanique aux transformations de la sphère productive. En premier lieu, il importe de ne pas penser la mondialisation comme une contrainte extérieure qui s'imposerait de façon inéluctable aux acteurs économiques : elle est aussi un cadre de pensée qui se propage dans les sphères économiques et dirigeantes, influençant ainsi les représentations des dirigeants d'écoles de commerce.

En second lieu, ce souci de l' « international » dans les ESC participe des logiques concurrentielles et des jeux de distinction entre ces établissements. En permettant à ces écoles de revendiquer une proximité avec les entreprises, en répondant à leurs besoins, l'« internationalisation " apparaît comme un élément de distinction vis-à-vis des formations universitaires à la gestion qui se créent à partir des années 1960 (Pavis, 2003b). Enfin, il s'agit pour l'ensemble des ESC de se démarquer des " petites " écoles qui se multiplient à partir des années 1970 et s'auto-proclament « internationales » : En s'appuyant sur l'Annuaire national des écoles de commerce et des formations supérieures à la gestion, publié par l'Étudiant en 1988, et qui indique la date de création des établissements, on constate que plus de 160 écoles de commerce sont créées en France entre 1967 et 1987 ; en outre, près d'un tiers d'entre elles comportent dans leur nom une référence à l'étranger : "international ", " européenne/European ", ou encore "American/américain ». Les responsables des ESC n'entendent pas laisser à ces " petites » écoles le monopole de l'international et ils s'emparent rapidement de cet enjeu (Lazuech, 1998). Des mécanismes d'imitation-distinction diffusent ainsi l'impératif de «l'internationalisation » dans les écoles de commerce qui, à partir des années 1980, en font un élément fort de leur identité.

À côté de ces préoccupations liées au contenu même de la formation, émerge également, dans les années 1980, l'idée que les écoles se doivent d'être "visibles » à l'échelle internationale. Tout un discours se construit progressivement dans le champ des formations à la ges.

5. A.-C. Wagner souligne qu'au début des années 1990 , la gestion est d'ailleurs le secteur d'enseignement supérieur où les accords avec les établissements étrangers sont les plus nombreux. Elle cite une étude de la FNEGE (Fondation nationale pour l'enseignement de la gestion des entreprises) qui montre qu'en 1992, $10 \%$ des étudiants en gestion ont suivi une partie de leurs études à l'étranger et que ce taux est plus élevé pour les écoles privées (12\%) que pour les étudiants à l'université (6 \%), (Wagner, 1997, p. 224). 
tion ${ }^{6}$ pour soutenir l'idée d'une concurrence à venir entre les formations européennes, en lien avec le développement du libre-échange et la construction de la CEE (communauté économique européenne). L'enjeu pour les écoles serait dès lors non seulement d'accéder à une certaine reconnaissance au-delà des frontières nationales mais aussi d'atteindre une " taille critique ». En effet, dans la majorité des pays européens, les formations à la gestion sont assurées au sein d'universités, dont les effectifs et les ressources sont sans commune mesure avec ceux des écoles de commerce françaises. C'est ainsi que les responsables des ESC justifient la mise en œuvre, dans la seconde moitié des années 1980, de "plans de développement " qui, sur un horizon de quelques années, définissent des objectifs en termes d'augmentation des effectifs étudiants, mais aussi d'accroissement du corps enseignant. A ce titre, l'exemple de l'ESC Lyon est révélateur : en 1987, le nouveau directeur, Bruno Dufour, lance un plan sur cinq ans dont l'objectif est de permettre à l'école de se forger une stature internationale : il s'agit d'atteindre un minimum de $10 \%$ d'étrangers parmi les enseignants, mais aussi parmi les étudiants, dont le nombre global doit aussi considérablement augmenter. En termes d'enseignement, les contenus doivent être " européanisés ", le nombre d'accords d'échanges doit augmenter afin de couvrir toute l'Europe, et il est prévu de mettre en place des cours bilingues?

Deux dimensions centrales du phénomène d' "internationalisation " des écoles de commerce ont été ici distinguées. La première dimension recouvre l'ensemble des mesures mises en place par les écoles pour "internationaliser " le cursus de formation (cours de langue, séjours à l'étranger, doubles-diplômes, etc.)... C'est d'abord dans cette première dimension que les ESC se sont internationalisées, avec une accélération du processus dans les années 1980. La seconde dimension recouvre les mesures destinées à accroître la « visibilité » des écoles. Si elle émerge dès les années 1980, ce n’est qu’à partir des années 2000 qu'elle devient prépondérante.

\subsection{La « visibilité internationale », un enjeu majeur dans les années 2000}

Cette seconde dimension du phénomène d'internationalisation devient, au cours des années 2000, un enjeu majeur pour les écoles de commerce en raison de la conjonction de plusieurs phénomènes. A l'échelle des ESC, l'enjeu est à la fois symbolique - le caractère international d'une école et sa reconnaissance hors des frontières se sont progressivement constitués comme preuves de son excellence - et stratégique. La reconnaissance internationale est en effet primordiale pour pouvoir établir des accords d'échanges, de doubles-

6.La Revue française de gestion, publiée par la FNEGE (Fondation nationale pour l'enseignement de la gestion des entreprises), consacre deux numéros, respectivement en 1989 ( $\left.\mathrm{n}^{\circ} 73\right)$ et en 1990 ( $\left.\mathrm{n}^{\circ} 78\right)$, aux enjeux de l'ouverture européenne pour les formations à la gestion.

7. source : entretien avec Bruno Dufour, archives de la Chambre de commerce et d'industrie de Paris : 813 W 47 : ESC Lyon : rapport d'activité 1988. 
diplômes et pour attirer des étudiants et enseignants étrangers, qui eux aussi concourent à la dimension internationale des formations dispensées.

Au-delà de ces enjeux propres aux écoles, l'Etat promeut, à la fin des années 1990, l'idée que l' "attractivité internationale » des institutions d'enseignement supérieur au sein d'un " marché éducatif " mondial constitue une priorité. Cet objectif est manifeste avec la création de l'agence EduFrance, en 1998, à l'initiative des ministres de l'Éducation nationale et des Affaires étrangères. La mise en place du processus de Bologne conforte, par la suite, cette vision selon laquelle les pays et leurs établissements d'enseignement supérieur sont désormais engagés dans une compétition pour attirer les meilleurs étudiants et enseignants-chercheurs.

Dans ce contexte, les ESC sont plus que jamais incitées à accroître leur visibilité hors des frontières. L'importance cruciale des enjeux liés à l'internationalisation se manifeste entre autres par la création généralisée de « directions internationales » et l'emploi de personnel à plein temps pour gérer les différentes missions liées à ces questions. Autre manifestation de l'enjeu que représente la reconnaissance internationale, plusieurs établissements adoptent une dénomination à consonance plus "internationale ", à l'instar de l'ESC Nantes qui devient, en 2000, Audencia, ou de l'ESC Marseille qui devient Euromed Marseille, en 2003, puis Euromed management, en 2008.

\subsection{Les accréditations, supports et moteurs de l'internationalisation}

C'est dans ce contexte d'une accélération du processus d'internationalisation qu'il convient d'appréhender un autre phénomène majeur, à savoir l'émergence des accréditations internationales dans le champ des ESC à la fin des années 1990. Une accréditation peut se définir comme un label décerné pour une durée déterminée par une organisation indépendante, non gouvernementale. Elle valide la conformité d'une institution d'enseignement supérieur dans son ensemble, ou d'un programme de formation en particulier, au regard de normes de qualité qu'elle a préalablement définies.

Au cours des années 2000, trois agences d'accréditation - l'EFMD (European foundation for management development), l'AACSB (Association to advance collegiate schools of business) et l'AMBA (Association of masters of business administration) - se sont imposées comme des acteurs de premier plan dans le champ des ESC ; en témoigne leur diffusion rapide auprès des écoles. Considérons les deux accréditations qui prennent en compte les établissements dans leur ensemble, et non pas uniquement un programme : l'accréditation EQUIS (European Quality Improvement System), proposée par l'EFMD, et l'accréditation AACSB, proposée par l'agence du même nom. En 2011, 19 établissements français 17 écoles, auxquelles d'ajoutent l'IAE (Institut d'administration des entreprises) d'Aix et l'université de Dauphine, étaient accrédités par la première, et 16 par la seconde. Parmi eux, 15 bénéficiaient d'une double accréditation. 
On peut s'interroger sur cet essor, étant donné le caractère volontaire du processus d'accréditation, mais aussi de son coût direct et indirect. De fait, la procédure est coûteuse : à titre d'exemple, dans la cas de l'ESC Toulouse, accréditée AACSB, EQUIS et AMBA, la chambre régionale des comptes de Midi-Pyrénées évalue que "sur la période 2005-2010, les coûts directs liés à ces accréditations, déplacements, cotisation, renouvellement, se sont élevés à $383000 €$ tandis que le coût du personnel directement engagé dans les processus de renouvellement, AMBA en 2007, EQUIS et AACSB en 2009, est estimé à $407000 € »^{8}$. A ces coûts financiers s'ajoutent les coûts liés aux changements parfois nécessaires dans le mode d'organisation de l'établissement. Ce responsable en charge des accréditations d'une " petite " ESC souligne ainsi que le processus d'obtention d'AACSB a nécessité de refondre les programmes pour les rendre plus cohérents et impliquera à terme un moindre recours aux enseignants vacataires : "cCest comme arrêter de prendre de l'hérö̈ne! Les vacataires, c'est peu coûteux, facile à manager, c'est très flexible, vraiment facile... mais pour l'AACSB, on doit changer" (entretien avec un responsable d'accréditation, 2010).

En dépit de ces coûts, le succès des accréditations s'explique par des logiques aussi bien nationales qu'internationales. Le fait que les premières écoles à avoir été accréditées soient les écoles généralement considérées comme les plus prestigieuses en France HEC, l'ESCP-EAP et l'EM (Ecole de Management) Lyon pour EQUIS et l'ESSEC pour AACSB - a contribué à en faire des symboles d'excellence, immédiatement recherchés par les autres écoles (Cret, 2007)9.

Du point de vue international, tous les responsables interrogés mettent en avant le fait que les accréditations participent à la visibilité des établissements et à leur reconnaissance au-delà des frontières hexagonales, aussi bien auprès des étudiants potentiels, des autres institutions de formation que des employeurs.

Le développement des accréditations apparait ainsi comme étroitement lié au processus d'internationalisation des ESC : tout en servant de support à une meilleure reconnaissance de ces établissements à l'étranger, les accréditations ont encore accentué la pression des écoles à "s'internationaliser", à travers des critères comme le recrutement d'étudiants étranger, l'existence de programmes d'échanges, mais aussi la présence d'enseignants étrangers ${ }^{10}$.

8. Chambre régionale des comptes de Midi-Pyrénées, Rapport d'observation définitive sur la gestion du groupe Ecole supérieure de commerce de Toulouse, exercices 2005 à 2009, 26 avril 2012 : p. 37; Rapportés aux dépenses totales, ces coûts financiers apparaissent néanmoins réduits dans ce cas : sur la période 20052012, les charges de l'ESC Toulouse s'élèvent à un total de 188 millions d'euros.

9. Soulignons que les accréditations peuvent aussi répondre à des logiques internes. B. Cret, qui a étudié l'accréditation de six établissements, dont trois en France, parle à ce titre d' "outil de gestion de second rang " et cite plusieurs exemples où les accréditations servent de justification à la direction, que ce soit pour supprimer un programme, ou pour gérer le personnel enseignant (B. Cret, op.cit. : pp. 215-260).

10. Les critères d'EQUIS et AACSB sont disponibles en ligne : http://www.efmd.org/index.php/accreditation-/equis/equis-documents et http://www.aacsb.edu/accreditation/standards.asp 
En se diffusant, ces agences ont ainsi participé à l'édiction de normes et critères d'évaluation des ESC, jouant ainsi le rôle de "juge de statut» (Sauder, 2005) dans la compétition qui les oppose. Outre les aspects relatifs à l' "international ", les accréditations ont joué un rôle majeur dans le "tournant académique " de ces écoles, c'est-à-dire l'essor sans précédent des activités de recherche en leur sein et une transformation rapide du corps enseignant.

\section{Le tournant académique 2 des Ecoles supérieures de commerce}

L'essor récent des activités de recherche dans les ESC peut surprendre, aussi bien par son ampleur que par sa rapidité. Certes, des écoles comme HEC, l'ESSEC ou l'EM Lyon ont initié ce type d'activité dès les années 1970, mais à une échelle très restreinte ; dans les ESC de province, la recherche reste, jusqu'à la fin des années 1990, une préoccupation extrêmement minoritaire. En ce sens, on peut effectivement parler d'un véritable tournant pour qualifier l'évolution à l'œuvre depuis une dizaine d'années : la recherche et surtout les publications dans des revues académiques sont devenues des activités centrales pour l'ensemble de ces écoles. Ce changement s'est accompagné d'une transformation du corps enseignant, celui-ci étant à la fois plus étoffé et plus diplômé.

\subsection{De la commission d'évaluation des formations au classement dans la presse}

Ce développement sans précédent des activités de recherche dans les écoles de commerce s'inscrit dans les transformations à l'œuvre dans le champ des écoles de commerce, à l'échelle nationale et internationale. En premier lieu, l'alignement de ces écoles sur le système LMD (licence, master, doctorat) ${ }^{11}$ et la mise en place d'une Commission d'Evaluation des Formations et Diplômes de Gestion (CEFDG) ont joué un rôle primordial dans l'essor de la recherche. Cette commission a été créée par le décret du 4 avril 2001, avec pour objectif d'exercer une "mission générale de contrôle de la qualité des formations des établissements consulaires et privés ${ }^{12}$. Composée de 16 membres issus du monde acadé-

11. Dans le cadre du processus dit de Bologne, de nouveaux diplômes sont créés en France : la licence $(b a c+3)$ et le master $(b a c+5)$. Les écoles de commerce peuvent apparaître a priori à l'écart de ce système, dans la mesure où elles recrutent traditionnellement plus au niveau bac +2 , notamment après deux années de classes préparatoires. Néanmoins, elles se sont alignées d'une certaine façon sur le système LMD ; d'une part, en développant un recrutement au niveau bac +3 ; d'autre part ,en transformant les deux dernières années de leur cursus en master.

12.Circulaire du 17 mais 2001 relative aux dispositions applicables aux établissements d'enseignement supérieur technique consulaires et privés relevant des articles L. 443-2 et L. 446-5 du code de l'Education et notamment aux écoles supérieures de commerce et et gestion. 
mique et professionnel, cette commission formule des avis sur le visa des diplômes délivrés par les écoles de commerce reconnues par l'Etat ${ }^{13}$ et, à partir de 2003, sur l'obtention du grade de master, un arrêté du 4 juin 2003 autorisant les écoles de commerce à demander ce diplôme.

La CEFDG a œuvré au développement de la recherche "académique " au sein des écoles de commerce, recherche qu'elle définit comme " celle qui entre dans le champ classique du domaine scientifique : communications dans les congrès et les colloques, publications dans les revues ${ }^{14 .}$ Cette recherche est définie comme une condition nécessaire pour obtenir le grade de master.

Deuxième facteur à considérer pour rendre compte du " tournant académique » des ESC françaises : les classements dans la presse. Ces classements ont en effet intégré les nouvelles exigences en matière de recherche, à l'instar de celui proposé par l'Etudiant qui introduit, en 2004, un critère portant sur le nombre de publications dans des revues listées par la section 37 (" économie et gestion ») du CNRS.

Dernier acteur ayant joué un rôle dans ce " tournant académique " des écoles de commerce en France : les agences d'accréditations. L'AACSB exige ainsi que $50 \%$ du corps professoral soit academically qualified, c'est-à-dire titulaire d'un doctorat et publiant régulièrement dans des revues à comité de lecture. Si aucun seuil n'est précisé pour obtenir l'accréditation EQUIS, les écoles candidates doivent néanmoins produire une description très détaillée des activités de recherche menées en leur sein.

Ainsi, qu'il s'agisse des normes édictées à l'échelle d'instances nationales, comme la CEFDG, ou internationales, comme les organismes d'accréditations, pouvoir mettre en avant une production scientifique réalisée par des chercheurs qualifiés apparait désormais nécessaire aux ESC pour acquérir une reconnaissance. En conséquence, ces écoles ont développé, depuis les années 2000 , des activités de recherche fortement orientées vers la publication dans les revues, puisque c'est à travers cet indicateur qu'elles sont évaluées. À ce titre, la définition que donnent les responsables de l'ESC Montpellier des activités de recherche imposées aux enseignants, est extrêmement révélatrice : «Par "recherche", il faut entendre les activités de production de savoirs menées selon une démarche scientifique faisant l'objet d'une publication retenue par le conseil scientifique. La réalisation d'un DEA (diplôme d'études approfondies), d'un DESS (diplôme d'études supérieures spécialisées), d'un master ou d'une thèse de doctorat n'est pas considérée comme une activité de recherche ${ }^{15}$.

13. Arrêté du 8 mars 2001 relatif aux diplômes délivrés par les établissements d'enseignement supérieur technique privés et consulaires reconnus par l'Etat. Auparavant, le visa était délivré une fois pour toute. A partir de 2001, il devient temporaire, avec une durée maximale de six ans.

14. Principes et orientation, Commission d'évaluation des formations et diplômes de gestion, 23 novembre 2005, p. 25

15. Sup de Co Montpellier, dossier de demande d'autorisation à délivrer le grade de master présenté par l'ESC Montpellier, Février 2004, p. 27. 
Cette orientation quasi-exclusive vers la publication témoigne d'une instrumentalisation de la recherche, qui apparaît avant tout comme un moyen d'acquérir un capital symbolique précieux dans un champ très compétitif. Mais ceci montre aussi que les écoles de commerce ont été influencées par des évolutions affectant de façon plus globale le monde universitaire, en l'occurrence la mise en place de classements de revues (Fridenson, 2009) et l'essor de la bibliométrie. Les écoles de commerce se sont emparées de cette nouvelle vision quantitative de la recherche, et ont fait leur le mot d'ordre " publish or perish".

\subsection{Comment les écoles se sont converties à la recherche académique}

Il n'est pas aisé d'objectiver la conversion des ESC à la recherche, dans la mesure où ce terme de " recherche " renvoie, pour ces établissements, à une pluralité d'activités - expertise, conseil, écriture de " cas " et de manuels, publications d'ouvrages et d'articles. Afin de justifier leur statut d'établissement de recherche, ces dernières se bornent généralement à afficher le nombre de leurs publications et à mettre en évidence leur augmentation. Si l'on s'en tient à cette approche " comptable ", on constate que dans toutes les ESC prises en compte ici, le nombre de publications a régulièrement augmenté depuis le début des années 2000 ; en témoignent les palmarès de L'Étudiant notamment qui comptabilisent, pour chaque école classée, le nombre d' " étoiles CNRS " des écoles classées ; ils considèrent ainsi, à partir du classement produit par la section "Economie et gestion " du CNRS, qu'un article publié dans une revue " trois étoiles " vaut trois articles publiés dans une revue à une étoile. Ainsi entre 2003 et $2007^{16}$, le nombre d' "étoiles " d'HEC est passé de 23 à 226, celui de l'ESCP de 4 à 112, ESC Toulouse 6 à 55, ESC Reims de 3 à 43, ESC Dijon de 1 à 20, ou encore ESC Amiens de 0 à 16.

Ainsi, les ESC aspirent désormais à être considérées comme des institutions de recherche à part entière et cherchent à concurrencer les universités dans ce domaine. Cette conversion à la recherche s'est effectuée à travers une redéfinition des missions attribuées aux enseignants, qui se sont vus contraints et/ou incités par des systèmes de prime à entreprendre des activités de recherche. L'essor de ces activités s'est par ailleurs appuyé sur une transformation du corps enseignant : le nombre d'enseignants permanents a augmenté dans toutes les écoles étudiées, ainsi que leur niveau de diplôme.

La transformation du corps enseignant s'est réalisée essentiellement par le recrutement de jeunes docteurs ou, pour les écoles qui en ont les moyens financiers, des "seniors, qui ont pris l'habitude de faire des étoiles ", pour reprendre les termes du directeur pédagogique d'une de ces écoles. Ces nouveaux enseignants à profil universitaire se distinguent de ceux recrutés pendant les années 1980 et 1990, qui étaient plutôt des professionnels, venus progressivement à l'enseignement, souvent titulaires d'un diplôme de 2e ou 3e cycle (DESS, diplôme

16. L'Étudiant $\mathrm{n}^{\circ} 268$, novembre 2004 et L'Étudiant, n 313 , novembre 2008. 
d'école de commerce). Le corps enseignant s'est donc non seulement étoffé et stabilisé, la proportion d'enseignants permanents par rapport aux vacataires s'étant accrue, mais aussi " professionnalisé ", dans le sens où il s'agit de plus en plus de personnes ayant été formées à l'enseignement et à la recherche. Ceci a eu pour effet de profondément transformer le marché du travail des enseignants-chercheurs en sciences de gestion, les écoles entrant en concurrence directe avec les universités mais aussi entre elles pour attirer les " publiants »; cela a eu pour effet d'accroître les dépenses liées aux activités de recherche : «Employer un bon professeur, ça coûte cher il faut donc payer - et déjà plus qu’à l'université - pour attirer les professeurs » explique un enseignant, responsable de la recherche dans une ESC.

Ce tournant académique résulte directement de la concurrence de statut qui oppose les écoles. Il apparaît relativement indépendant de la « demande » des étudiants ou des entreprises : les étudiants ne se préoccupent guère des publications classées par le CNRS, et de la même façon, les recruteurs valoriseront plus l'expérience professionnelle acquise dans les stages ou les séjours à l'étranger que le fait d'avoir suivi un cours avec tel enseignant "publiant ". Dès lors, un des enjeux pour les responsables d'établissement consiste à concilier les activités de recherche avec l'image d'écoles professionnelles qu'ils cherchent à promouvoir auprès des étudiants potentiels et de la sphère économique.

Dans les faits, c'est surtout par un découplage entre les activités d'enseignement et de recherche que les écoles parviennent à concilier leur double identité. Les politiques de gestion du personnel enseignant contribuent en effet à cloisonner les activités de recherche et d'enseignement au sein des ESC : toutes les écoles étudiées ont mis en place des grilles d'évaluation intégrant des objectifs en termes de recherche et d'enseignement différenciés en fonction de "profils" ou de " parcours".

\subsection{Financer la recherche, rechercher des financements}

Le développement des activités de recherche et les transformations du corps enseignant des ESC posent des problèmes en matière d'identité institutionnelle mais aussi et surtout en termes de financement. Comment financer les activités de recherche? Où trouver les fonds pour recruter les enseignants-chercheurs les plus " productifs" ?

La dimension financière est de fait centrale. En témoigne la corrélation entre le budget des écoles et le nombre de publication, corrélation médiatisée par la taille des établissements et les effectifs du corps enseignant ( $c f$. Figure 1). 
Figure 1. Relation entre le budget et la production scientifique des écoles de commerce

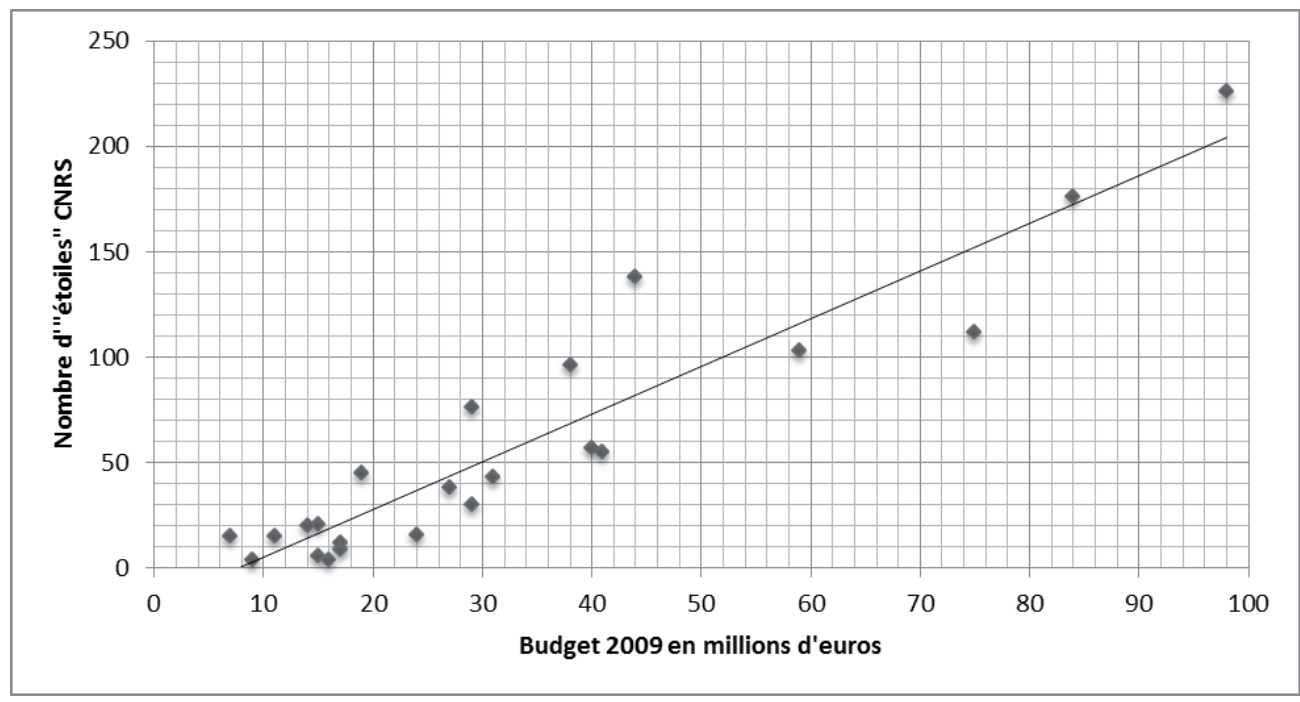

$r=0,93, r 2=0,87$

Commentaire : À partir des données de budget disponibles dans la revue Challenges et du nombre d' « étoiles » comptabilisées en 2007-2008 par le magazine L'Étudiant pour 26 écoles, on peut établir une corrélation linéaire entre ces deux séries de données, ce qui montre bien l'impact du facteur financier pour les publications.

Sources : Challenges, $n^{\circ} 193$, décembre 2010, et L'Étudiant, n³13, novembre 2008.

Les budgets globaux et les montants investis dans la recherche et dans le recrutement des enseignants sont extrêmement variables entre les ESC ; cependant, toutes ont dû trouver un moyen d'augmenter leurs ressources afin de faire face aux nouvelles dépenses liées au tournant académique. Diverses techniques ont été mises en œuvre, comme la création de fondations ou de chaires. Néanmoins, ce sont essentiellement la hausse des frais de scolarité et l'accroissement des effectifs qui ont permis aux écoles de dégager les budgets nécessaires à l'essor des activités de recherche. Comme le rappelle le récent rapport de la Cours des comptes ${ }^{17}$, en l'espace de 4 ans (rentrée 2007 à rentrée 2011), les effectifs en formation initiale des écoles de commerce consulaires ont augmenté de $39 \%$, tandis que pour 19 ESC, la moyenne hors inflation des frais de scolarité sur trois ans du programme dit "Grande Ecole " (programme permettant d'obtenir un

17. Cour des comptes, Les écoles supérieures de commerce et de gestion : un développement à réguler, rapport public annuel, 2013. 
master) a augmenté de 28,6 \% entre 1999 et 2010. Dans le même temps, les effectifs ont fortement augmenté, en lien avec une transformation du recrutement et de l'offre de formation.

\section{Des grandes écoles aux grands groupes: 2. la massification des ESC}

Comme nous l'avons souligné en introduction, les écoles de commerce françaises, et parmi elles les ESC, ont connu une croissance particulièrement rapide au cours de la décennie passée. Comment peut-on rapprocher cette évolution des deux précédentes, et plus généralement de la concurrence de statut qui oppose les ESC ?

Ces stratégies sont développées à partir des années 1980, ce qui constitue une rupture par rapport aux logiques anciennes : en effet, alors que la plupart des ESC étaient auparavant essentiellement dans une logique de survie, s'impose désormais une logique de croissance. Les responsables des écoles cherchent dès lors à attirer un nombre toujours plus important d'étudiants et à conquérir des "parts de marché » afin d'accroître leurs ressources financières - et soutenir l'internationalisation et l'académisation - mais aussi leur visibilité et donc leur statut.

Deux types de stratégies sont mis en œuvre. D’une part, ces écoles augmentent la taille des promotions de leur programme le plus ancien, c'est-à-dire la formation en trois années accessible entre autre après une classe préparatoire, que l'on retrouve généralement sous le vocable de " programme Grande Ecole». D'autre part, les ESC se développent en diversifiant leur offre de formation à travers la création de nouveaux programmes.

Loin de constituer une simple réponse à des demandes sur le marché de la formation ou du travail, le très fort accroissement du nombre d'étudiants des ESC résulte aussi de stratégies de développement clairement définies par les dirigeants de ces écoles. Certes, il importe ainsi, à la suite de J.-P. Briand et J.-M. Chapoulie (1993), de s'intéresser à l'offre de places des écoles et aux modalités institutionnelles de son augmentation. Comme l'ont montré les deux auteurs dans le cas de l'instruction primaire, c'est en référence à ce qu'une institution d'enseignement donnée offre en termes de places qu'une demande de la part des familles ou des entreprises ${ }^{18}$ peut se constituer. En mettant en avant cette notion d' "offre de places ", ils insistent donc sur le rôle des institutions par rapport à la "demande sociale d'éducation ".

18. Ibidem : p. 12. 


\subsection{Accroitre la taille des promotions au sein du « programme Grande Ecole »}

Le programme dit "Grande Ecole " constitue le premier levier de croissance des ESC. A partir des années 1980, le nombre de places offertes au concours pour les étudiants candidats à ce programme, issus de classes préparatoires commerciales, va régulièrement augmenter ( $c f$. Figure 2).

Figure 2. Évolution des places ouvertes aux concours des ESC pour les étudiants de classes préparatoires entre 1985 et 2002

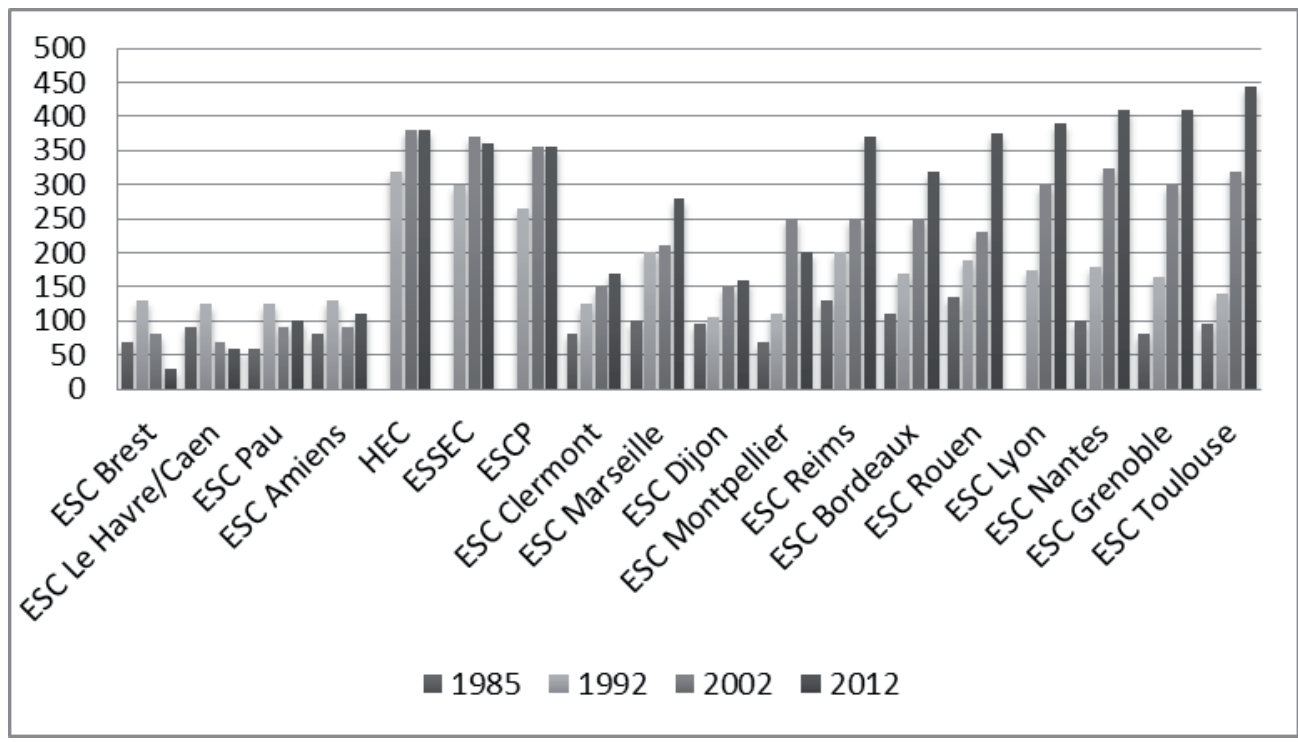

Lecture : Entre 1985 et 2012, le nombre de places offertes au concours d'entrée l'ESC Rouen aux étudiants issus de classes préparatoires est passé de 135 à 375 .

Commentaire : On note une augmentation des places offertes dans toutes les écoles entre 1985 et 1992. La tendance se diversifie ensuite : alors que certaines « petites » écoles (ESC Brest ou Pau) offrent un nombre de place qui diminue, ce qui témoigne d'une réorientation du recrutement vers les admissions parallèles (voir ci-dessous), ce nombre stagne dans la dernière décennie pour les écoles parisiennes (HEC, ESSEC, ESCP) et continue de croitre fortement pour un certain nombre d'ESC de province.

Source : Graphique réalisé à partir de données du ministère de l'Education nationale (données non disponibles pour HEC, I'ESSEC l'ESC Lyon et l'ESCP en 1985).

Néanmoins, le vivier des classes préparatoires peine à assurer une croissance régulière des ESC et les admissions dites " parallèles » ou " sur titre » vont constituer un autre moyen de développer le programme "Grande Ecole ». Ces admissions sont ouvertes aux étudiants n'ayant pas suivi une classe préparatoire et titulaires d'un diplôme de l'enseignement supérieur (bac +2 ou bac +3$)$. Apparu dès la fin des années 1960, ce mode de recrutement reste cependant relativement marginal jusqu'aux années 1980 : au concours de 1980, les candidats recrutés de cette façon ne représentent que $8 \%$ des admis dans les ESC de province. Il va progressivement se développer au cours de cette décennie et connaître une 
croissance rapide dans les années 1990, alors que le nombre d'étudiants en classes préparatoires commerciales connaît une légère décru, phénomène accentué par le passage de cette préparatoire à deux années en $1995^{19}$.

Alors même que les effectifs en classe préparatoire commerciale se stabilisent puis augmentent fortement après 2005 (+ 21\% entre 2005 et 2010), l'essor des admissions parallèles se poursuit au cours des dernières années, à la fois pour soutenir la croissance des écoles, mais aussi pour permettre une certaine harmonisation avec le système d'enseignement supérieur européen (LMD), en développant les recrutements à bac +3 . Les étudiants intègrent ainsi directement la 2e année du programme, pour préparer un master en management.

Figure 3. Évolution des places offertes aux concours dans 22 ESC entre 2001 et 2009

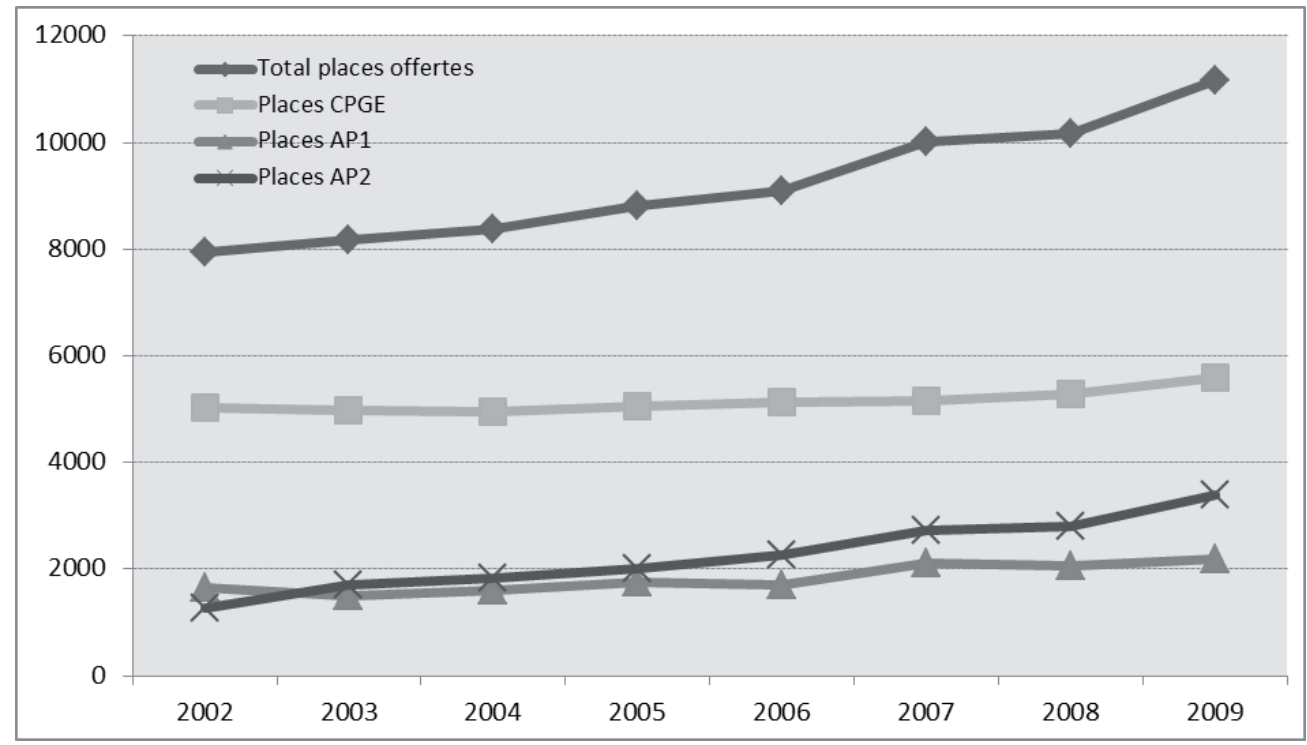

*AP1 : Admissions parallèles 1 : admissions parallèles au niveau bac +2 pour entrer en première année du programme "Grande Ecole ", AP2 : Admissions parallèles 2 : admissions parallèles au niveau bac +3 pour entrer en deuxième année du programme « grande Ecole ». CPGE : Classes préparatoires aux grandes écoles.

Source : Graphique réaliséa à partir du nombre de places offertes annoncées dans les bulletins officiels publiés entre 2001 et 2009 pour les établissements suivants : ESC de Toulouse, Reims, Rouen, Nantes, Lyon, Bordeaux, Marseille, Grenoble, Tours-Poitiers, ESCP, ESSEC, HEC, ESC Lille, ESC de Clermont, Dijon, Nice, Montpellier, Pau, La Rochelle, Amiens, Brest, Le Havre-Caen.

19. Elle était en effet, à la différence des classes scientifiques et littéraires, d'une année jusqu’à cette date. 
En conséquence, la proportion d'étudiants fréquentant le programme «Grande Ecole » et issus des classes préparatoires tend à diminuer (Figure 3). Ce point mérite d'être souligné, alors que les débats récents sur l' " ouverture sociale » des grandes écoles en France se sont largement focalisés sur les classes préparatoires, et que les discours politiques et médiatiques aussi bien qu'académiques ont occulté la question des admissions parallèles.

Concernant l'origine scolaire des étudiants entrés par cette voie, il est difficile d'obtenir des données précises. Les chiffres transmis par le service communication du concours Passerelle indiquent que, en 2007, près de $80 \%$ des candidats et des étudiants intégrés en AP1 étaient issus d'un cursus spécialisé en commerce, économie-gestion ou gestion-comptabilité. Ainsi, le passage par une école de commerce apparaît majoritairement lié à la formation passée, contribuant à faire des cursus en gestion de niveau $\mathrm{bac}+2$, un vivier pour le recrutement du programme "Grande Ecole » des ESC. En AP2, ce constat reste vrai, même si l'on note une légère diversification : les étudiants issus d'un cursus en commerce, économie-gestion ou gestion-comptabilité, représentent toujours $70 \%$ des admis, mais $7 \%$ des intégrés sont issus d'un cursus en langues, et $5 \%$ en droit. Ces données relativisent le discours des écoles justifiant le recours croissant aux admissions parallèles par la volonté de diversifier le profil et les expériences de leurs étudiants

\subsection{Diversifier l'offre de formation}

La forte croissance des ESC depuis une dizaine d'année s'appuie en outre sur la diversification des programmes. En élargissant leur offre de formation, les écoles peuvent atteindre un public plus divers que celui des seules classes préparatoires En outre, certains programmes dégagent des profits permettant de soutenir le développement de l'ensemble de l'établissement.

Si les ESC ont très tôt proposé des mentions ou des programmes complémentaires, ce n'est qu'à partir des années 1980 que ce phénomène commence à prendre une réelle ampleur, avec l'essor de la formation continue, l'apparition de programmes post-bac et surtout la création des " mastères spécialisés ". Créé en 1986 par la Conférence des grandes écoles (CGE), et réservé aux membres de cette association, ce label valide des formations de courtes durées (entre un an et un an et demi), accessibles sans passer par le filtre du concours. Il a été à l'origine conçu par les écoles d'ingénieurs et destiné aux étudiants étrangers, dont il vise à sanctionner les séjours d'échanges dans des écoles françaises. Ce sont pourtant essentiellement des étudiants venus des universités françaises ou d'écoles de commerce moins cotées qui se sont d'abord tournés vers ces programmes. A titre d'exemple, à Nantes, dès 1992, les étudiants des mastères spécialisés viennent à $53 \%$ de l'université, les autres étant issus à $40 \%$ d'écoles de commerce, $7 \%$ d'écoles d'ingénieurs et $10 \%$ possédant une expérience professionnelle ${ }^{20}$.

20. Comité national d'évaluation des établissements publics à caractère scientifique, culturel et professionnel, 
Au cours de la dernière décennie, ce phénomène de diversification de l'offre s'est accéléré, notamment avec l'essor de programmes en trois ans, dits "programmes bachelor ", accessibles sur concours pour les titulaires du baccalauréat. Comment comprendre l'émergence généralisée de ces programmes ? Sous l'effet de la pression concurrentielle, les établissements tendent à s'aligner de façon consciente sur les pratiques " efficaces » mises en place par leurs concurrents, comme l'explique ce responsable du programme "Grande Ecole » d'une ESC : "Très souvent, on fait appel à des consultants extérieurs qui sont missionnés sur des choses très ponctuelles, le lancement d'un nouveau programme, sur une veille internationale. On s'est doté depuis deux ans d'une personne qui est chargée de faire des mini études concurrentielles. Mais quand on veut aller plus loin, il faut avoir recours à des cabinets extérieurs. On a des sociétés externes qui nous vendent des offres pour nous conseiller sur nos stratégies. " Ces pratiques ont été mentionnées par plusieurs enquêtés qui, de façon générale, mettent en évidence un effort de veille sur les autres institutions : "On a une veille permanente de ce qui est fait dans les autres écoles, et pas seulement en France, mais aussi à l'international" résume ainsi cet enseignant d'une autre ESC.

Les responsables des ESC ont vite pris conscience de l'atout que constituaient les programmes de type «bachelor ». Ces programmes permettent tout d'abord aux écoles de s'aligner sur le système LMD, progressivement mis en place dans les universités françaises à partir de 2003, et ainsi de mieux s'insérer dans le système d'enseignement supérieur national et européen. Ces programmes sont en outre présentés comme ouvrant un accès direct au marché de l'emploi mais aussi comme une première étape avant le master, ce qui permet d'attirer à la fois les étudiants envisageant des études courtes, et d'autres des études plus longues.

Enfin, les programmes "bachelors " représentent un moyen pour ces écoles de poursuivre leur croissance numérique et de dégager des ressources, alors que le potentiel de développement de leurs programmes "Grande Ecole " peut apparaître plus limité sur le long terme. Une étude de l'ESC Toulouse, menée par la chambre régionale des comptes, montre ainsi que si ces deux programmes dégagent des bénéfices nets, entre 2005 et 2010, ils ont augmenté de $186 \%$ pour le programme « Grande Ecole » et de $227 \%$ pour le programme " bachelor $»^{21}$.

Ce phénomène de diversification de l'offre qui touche l'ensemble des ESC est difficile à quantifier en raison de la pauvreté des sources. Néanmoins, il semble que la diminution de la proportion d'étudiants inscrits dans le programme "grande école " tende à se généraliser : les données du ministère de l'Education nationale montrent une diminution, entre 2001 et 2009, de la part des diplômes visés (donc du diplôme "grande école " pour les ESC) parmi les diplômes délivrés par les écoles de commerce reconnues

1992, Le Groupe ESC Nantes-Atlantique, rapport d'évaluation, Paris : Comité national d'évaluation. 21. Chambre régionale des comptes de Midi-Pyrénnées, 2012, op.cit. : p. 44. 
par l'Etat et délivrant un diplômé visé : cette proportion passe de 80 à $68 \%$, au profit des autres diplômes ${ }^{22}$.

Ce constat invite là aussi à reconsidérer d'une part, l'image souvent admise de la "grande école " à la française qui offre une formation en trois année après une classe préparatoire ; d'autre part, à nuancer le clivage qui peut exister entre le système universitaire et celui des écoles, puisque les étudiants peuvent circuler entre les deux et intégrer une école après une formation universitaire. Enfin, il appelle à nuancer les approches trop globalisantes sur les écoles de commerce, qui tendent à les penser comme un tout homogène, alors que les profils sociaux et scolaires des publics ainsi que leur devenir professionnel ne sont pas nécessairement les mêmes dans les différents programmes proposés ${ }^{23}$.

\section{Conclusion}

Cet article a pris comme point de départ la croissance qu'ont connue les ESC, et plus généralement les écoles de commerce, au cours des dernières décennies, et le poids de plus en plus prégnant de ces établissements dans l'enseignement supérieur. Cet essor des écoles de commerce doit être rapproché d'autres évolutions dans le champ de l'enseignement supérieur, comme la désaffection relative dont souffre l'Université, et les transformations dans les modalités de choix d'orientation des étudiants. Comparant les résultats obtenus lors de l'enquête "Conditions de vie des étudiants " de l'OVE (Observatoire de la vie étudiante) de 2010 à ceux des années antérieures, Philippe Cordazzo (2011) montre ainsi que "l'attractivité d'une filière tend aussi et de plus en plus à être évaluée à l'aune de critères ayant trait à l'insertion professionnelle quelle procure à ses diplômés». On assiste de fait à un essor rapide des formations dites "professionnelles ", auquel participent les écoles de commerce. Ceci est d'autant plus vrai que les évolutions du marché du travail contribuent à rendre ces formations attractives en offrant à leurs diplômés des chances relativement élevées d'occuper un emploi de cadre : ainsi $65 \%$ des personnes qui ont obtenu leur diplôme d'écoles de commerce en 2004 sont cadres en 2007 (Calmand et al., 2009). En cela, le poids acquis par les écoles de commerce peut se lire comme un véritable fait social, résultant de tendances « lourdes».

Notre propos ici était de mettre en évidence la façon dont la concurrence de statut qui oppose les ESC contribue finalement à façonner leur évolution. Ainsi les transformations qui ont affecté des établissements au cours des dernières décennies ne reflètent pas nécessairement directement les attentes des familles ou des entreprises en termes d'insertion professionnelle ; cela remet donc en cause l'image de secteurs de formations directement

22. Tableaux statistiques, juin 2002, «Les effectifs d'établissements privés d'enseignement supérieur non universitaires de commerce et de gestion » et Note d'information, décembre 2010, «Les étudiants dans les écoles de management reconnues à diplôme visé en 2009-2010. Augmentation continue des effectifs ».

23. Les éléments recueillis en entretiens semblent aller dans ce sens ; néanmoins, il n'existe pas de données disponibles croisant l'origine scolaire - et a fortiori sociale - des étudiants en fonction des programmes. 
en prises avec les demandes qui leur sont adressées, et notamment celles du monde du travail.

\section{Bibliographie}

Blanchard M. (2012), Socio-histoire d'une entreprise éducative : le développement des Ecoles supérieures de commerce en France (fin du XIXe siècle-2010), Thèse de doctorat en sociologie, EHESS.

Bourdieu P. (1989), La Noblesse d'État, Paris, Les Éditions de Minuit.

Briand J.-P et Chapoulie J.-M. (1993), "L'institution scolaire et la scolarisation : une perspective d'ensemble ", Revue française de sociologie vol. 34, $\mathrm{n}^{\circ}$ 1, p. 3-42.

Calmand J., Epiphane D. et Hallier P. (2009), "De l'enseignement supérieur à l'emploi : voies rapides et chemins de traverse. Enquête "Génération 2004", interrogation 2007 ", Notes Emploi Formation, Céreq, n 43, octobre.

Cordazzo P. (2011), "Choix d'orientations, quelles réalités ? » in Galland O., Verley E. et Vourc'h R. (s.d.) Les mondes étudiants. Enquête condition de vie 2011, La Documentation française, Paris, p.95

Cret B. (2007), L'émergence des accréditations : origine et efficacité d'un label, Thèse de doctorat, IEP de Paris.

DiMaggio P. et Powell Walter (1983), "The iron cage revisited: institutional isomorphism and collective rationality in organizational fields ", American Sociological Review vol. 48, n 2, pp. 147-160.

Fridenson P. (2009), "La multiplication des classements de revues de sciences sociales ", Le Mouvement social, $\mathrm{n}^{\circ} 226$, pp. 5-14.

Karpik L. (1989), "L'économie de la qualité », Revue française de sociologie, vol. 30, $\mathrm{n}^{\circ} 2$, pp. 187-210.

Lazuech G. (1998), "Le processus d'internationalisation des grandes écoles françaises ", Actes de la recherche en sciences sociales, vol. 121, n ${ }^{\circ}$, pp. 66-76.

Pavis F. (2003a), Sociologie d'une discipline hétéronome. Le monde des formations en gestion entre universités et entreprises en France. Années 1960-1990, Thèse de sociologie, université Paris I.

Pavis F. (2003b), «L'institutionnalisation universitaire de l'enseignement de gestion en France. (1965-1975)", Formation Emploi, n 83, pp. 51-63.

Saint-Martin M. (de) (1997), "Les écoles de gestion : un espace de concurrence en crise ? ", in de Saint-Martin M. et Gheorghiu M.-D. (s.d), Les écoles de gestion et 
la formation des élites, Actes du Colloque à la Maison Suger, 10-11 octobre 1996, pp. 3-12.

Sauder M. (2005), Status judges: the effects of formal rankings on educational status systems, Thèse de doctorat, Northwestern university.

Wagner A.-C (1997), "Les écoles de commerce et la gestion d'une culture internationale ", in de Saint-Martin M. et Gheorghiu M.-D. (s.d), Les écoles de gestion et la formation des élites, Actes du Colloque à la Maison Suger, 10-11 octobre 1996, pp. 223-236.

White H. (1981), "Where do markets come from?", American Journal of Sociology, vol. $87, \mathrm{n}^{\circ} 3$, pp. 517-547. 